\title{
Bulletin critique
}

\section{'Alqama, Hidāš b. Zuhayr et Ṭa’abbața Šarran}

Le cédrat, la jument et la goule : trois poèmes préislamiques, trad. Pierre Larcher, Arles, Sindbad-Actes Sud, 2016, 93 p., IS BN : 978-2-330-06989-6, $16 €$ broché.

Plus je traduis de poèmes et plus je pense que cette poésie et les "histoires" qui lui sont associées consignent moins l'histoire des Arabes que leur mythologie : malheureusement, dans le monde de l'islam, qui n'a pas fait sa révolution herméneutique, la théologie a tué la mythologie. Quant aux arabisants, ils manquent de l'audace ou, plus simplement, des compétences nécessaires ${ }^{1}$.

Tel est le bilan, assez sombre, que Pierre Larcher dressait des études contemporaines sur la poésie préislamique dans une conversation récente portant sur ce sujet, et aussi sur sa « courbe de vie », qui y est depuis des décennies si étroitement liée. Toutefois, s'il dénonce un manque, c'est parce qu'il s'est engagé depuis longtemps à y remédier, non pas par des élucubrations théoriques, mais dans la confrontation avec des textes vivants, dont le secret ne se livre qu'après une patiente recherche linguistique sur les mots et sur ce qu'ils signifi(ai)ent. Le cédrat, la jument et la goule, dernier fruit de cette activité à la fois de traduction et d'analyse, avance décidément dans le sens d'une lecture mythologique.

Parmi les trois essais qui composent le volume, « La goule » est probablement le plus audacieux. Présenté à une journée d'études qui s'est déroulée en avril 2015 à Naples, il traduit un poème (un autre étant donné en annexe) de Ta’abbața Šarran, où ce héros préislamique raconte, pour emprunter les mots à Ibn Qutayba (m. 276/889), qu'« il avait rencontré la goule et l'avait tuée ». Et Pierre Larcher de suivre les traces de ce cryptide assimilé à notre ogresse,

1 «Actualité de la poésie préislamique : conversation avec Pierre Larcher, propos recueillis par Martino Diez », Oasis Longreads, 1 (2017), http://www.oasiscenter.eu/fr/newsletter/2017/o2/ 16/actualit\% $\%$ 3\%A9-de-la-po\% $\mathrm{C}_{3} \%$ Agsie-pr\% $\% \mathrm{C}_{3} \%$ Agislamique-longreads-n-1-2017, consulté le 23 juin 2017. 
masculin quant à la forme, féminin quant au sens, qu'il met en relation avec le mythe de Persée et de Méduse. Si la voie mythologique avait été déjà essayée par Suzanne Pinckney Stetkevych, qui avait vu dans le texte une allusion au Sphinx, la connexion proposée par Pierre Larcher se fait par le biais de l'astronomie arabe, où la constellation de Persée est appelée « porteur de la tête de la goule ». Cette «transculturation révélatrice» (p. 68) se manifeste également dans la direction inverse, car une des étoiles de cette constellation porte, en Occident, le nom d'Algol, issu justement de l'arabe al-gūl. Autant d'éléments extratextuels qui, unis à l'analyse linguistique, amènent Larcher à conclure que le bref poème de Ta'abbața Šarran serait la version arabe du mythe de Persée, « condensé » et inséré dans le cadre de la qașida. En particulier, le mythe arabe aurait en commun avec son homologue grec la corrélation à la sexualité masculine, « avec ses pertes et ses doutes» (p. 71). Le pas vers une lecture mythologique, qui s'annonçait au lecteur au moins depuis Zuhaira! $!^{2}$, est donc bel et bien franchi.

Par contre, dans le second essai, « La jument », il n'est pas question de mythologie, mais d'anthropologie. Larcher traduit un poème d'un auteur mineur, la muğamhara de Huidāš b. Zuhayr, qui se caractérise par « sa brièveté, sa structure et son obscurité » (p. 46). En s'appuyant sur le habar du poème, il le lit comme « l'histoire d'un conflit entre deux types de solidarité, l'une fondée sur la parenté par le sang, l'une fondée sur une alliance » (p. 49). Passionné d'opéra, Larcher, lui, a autrefois comparé la production poétique préislamique, les deux formes d'art ayant en commun l'existence d'un répertoire plus ou moins fixé. Dès que l'on sort de celui-ci, comme c'est le cas avec Huidāš b. Zuhayr, on se retrouve dans une terre largement inconnue, où l'interprète doit faire appel à sa sagacité pour s'en tirer avec profit. La thèse de Larcher est que, surtout dans les cas où les commentaires se taisent ou se révèlent insuffisants, il est impératif de mettre en valeur le habar, en le considérant comme la clé symbolique qui permet de lire de façon cohérente et non arbitraire les allusions éparses dans les vers.

Mais c'est le poème en mìm de 'Alqama, « Le cédrat », quil'emporte surle plan artistique. Lauteur y est conduit par l'appellation de «treizième Mu'allaqa » qui lui est parfois décernée. Larcher montre la genèse de l'erreur, dont la faute revient à Ibn Hुaldūn (m. 808/1406) même. L'historien maghrébin aurait croisé les auteurs des Six poésies, collection particulièrement prisée dans l'Occident musulman, avec la liste des auteurs des Múallaqāt donnée par Ibn Rašīq

2 Abū Kabīr al-Hudalāī, Zuhayra! Quatre poèmes à sa fille sur la vieillesse et la mort, trad. Pierre Larcher, Arles, Sindbad-Actes Sud (« La petite bibliothèque de Sindbad »), 2014. Compte rendu de cet ouvrage par le même auteur publié dans Arabica, 62/4 (2015), p. 587-589. 
(m. 456/1063-1064 ou 463/1070-1071) dans al-'Umda fi șin'at al-ši'r, et qui diffère de la version commune des Sept poèmes. Mais « si ce poème n'est pas une Múallaqa, il pourraitl'être »(p. 21). Dansla conversation citée plus haut, Larcher, sollicité de donner son « hit-parade » personnel de la poésie préislamique, avouait, en effet, que « souvent, quand la famille s'agrandit, la préférence va au petit dernier. Aussi citerai-je comme aperçu de cette parole poétique quelques vers extraits de ma dernière traduction $[\ldots]$ : “Oui, je suis monté sur la selle arçonnée, hâlé par / Une journée, que les Gémeaux apportent, empoisonnée / Brûlante, comme si l'ardeur du feu l'enveloppait / Malgré les vêtements, tête de l'homme enturbannée"... Il s'agit d'une course dans le désert, sur une chamelle, aux premières chaleurs, apportées par un vent du Sud, qu'on croit chargé de miasmes ».

Dans la traduction du poème de Alqama, la tension linguistique que Larcher considère comme une constante de la production poétique arabe touche à son apex, en aboutissant à des résultats qui sont à la fois archaïques dans le fond et neufs (et convaincants) dans leur forme. Un seul exemple suffira : «Au rappel de Salmâ! Me rappeler le temps avec / elle ? Simple sottise! Supputer l'absence? Lithomancie! » (v. 13, p. 27).

Lecture mythologique-anthropologique et tension hermétique représentent ainsi les deux points d'arrivée, provisoires, de la conversation que Pierre Larcher poursuit depuis plus de quarante ans avec la poésie arabe préislamique. Cette parole fonctionne, de l'aveu même de l'auteur, «comme un plus, ou, plutôt, comme la philosophie pour Boèce : une consolation ». Pour Larcher certainement; mais non moins pour ses lecteurs.

\section{Martino Diez}

Université Catholique de Milan martino.diez@unicatt.it 